\title{
Una historia de dos bosques: el ocaso de la vegetación subtropical Afro-Macaronésica
}

\begin{abstract}
Este artículo es una versión del artículo galardonado en la segunda edición de los Premios César Gómez Campo en la categoría mejor trabajo científico:

- Mairal, M., J. Caujapé-Castells, L. Pellissier, R. Jaén-Molina, N. Álvarez, M. Heuertz \& I. Sanmartín (2018). A tale of two forests: ongoing aridification drives population decline and genetic diversity loss at continental scale in Afro-Macaronesian evergreen-forest archipelago endemics. Annals of Botany 122: 1005-1017.
\end{abstract}

Cuesta imaginar que hace tan solo siete millones de años, cuando el Mioceno estaba llegando a su fin, el norte de África era un territorio húmedo, donde se extendía una vegetación de carácter subtropical. Sin embargo, un intenso periodo de actividad tectónica estaba a punto de comenzar: la Placa Africana se estaba elevando en su parte este, en un proceso que iba a formar los actuales arcos montañosos en torno al Gran Valle del Rift (Figura 1). Este levantamiento topográfico creó una gran sombra de lluvia orográfica, que dio lugar a los primeros signos de aridificación en el centro y oeste del continente. Posteriormente, esto se vio potenciado con la llegada del Plioceno Medio (hace 3.5 millones de años), periodo que iba a desencadenar un gran calentamiento global, afectando profundamente a las floras al sudoeste del Paleártico.

En su conjunto, todos estos cambios provocaron una enorme aridificación, lenta y gradual, que obligó a que muchas especies migraran hacia hábitats similares a los de sus nichos climáticos, fragmentaran sus poblaciones o se extinguieran. El resultado fue la extirpación de numerosas especies en el norte de África y la formación del desierto del Sáhara (Axelrod $\&$ Raven 1978). Pero muchas de esas especies no se extinguieron totalmente, sino que algunos de sus representantes migraron a refugios climáticos en los bordes del continente e islas adyacentes: áreas con un carácter más húmedo, debido a su topografía (p. ej. cordillera del Atlas y macizo Etíope) o a la influencia de un suave clima oceánico (p. ej. Islas Canarias, Cabo Verde y Archipiélago de Socotra; Figura 1).
DOI: $10.15366 /$ cv2019.23.004

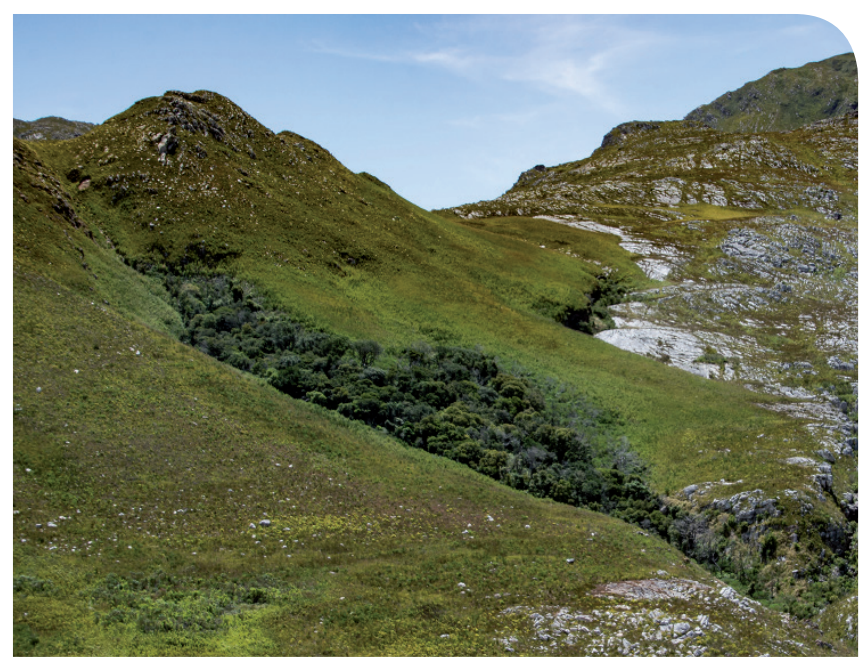

Retazo de bosque Afromontano.

(Foto Mario Mairal).

De esta manera, linajes hermanos quedaron aislados en ambos extremos del continente africano en un fenómeno conocido como vicarianza ecológica. A su vez, y debido a que la gran aridificación de África fue gradual, los linajes no migraron súbitamente, sino que siguieron una secuencia temporal que se ajustó a su nicho climático. Es decir, los taxones de carácter subtropical, más sensibles a la aridez, fueron los primeros en desplazarse, mientras que los taxones xerofíticos, con mayor tolerancia a ambientes secos, resistieron durante más tiempo (Mairal et al. 2017a). Así, muchas de las especies que antes estaban ampliamente distribuidas se convirtieron en especies relictas - especies raras con distribución reducida- y en paleo-endemismos —especies relictas que han sufrido una amplia extinción en su rango de distribución original-. Estos paleo-endemismos son los únicos supervivientes de ramas del árbol de la vida muy "podadas" (linajes reduci- 
dos por la pérdida de biodiversidad asociada a extinciones), considerados a menudo como fósiles vivientes y, por tanto, representantes de una biodiversidad única y evolutivamente antigua, de especial prioridad a nivel conservacionista.

Todavía hoy podemos encontrar testigos de esta larga crónica evolutiva en las floras de África del Norte si, por ejemplo, hacemos una incursión botánica por algún paraje de las Islas Canarias. Aquí crecen varios géneros (p. ej. Aeonium, Campylanthus, Camptoloma, Kleinia, Plocama), cuyos linajes hermanos

a)

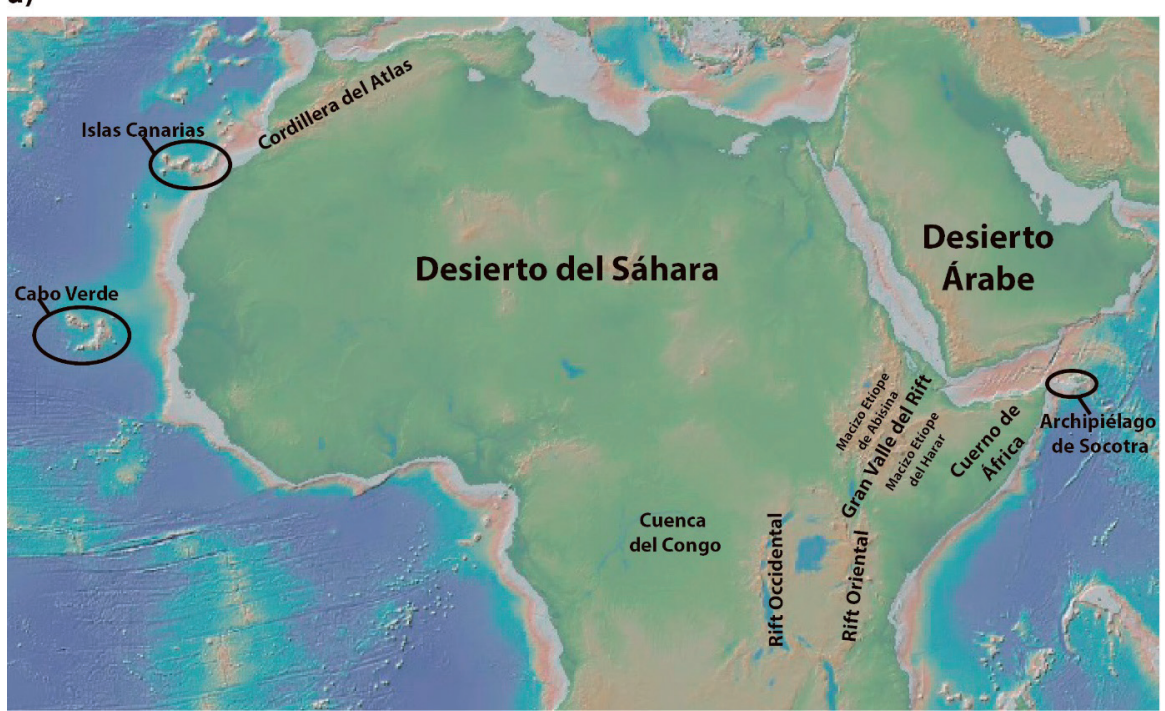

b)
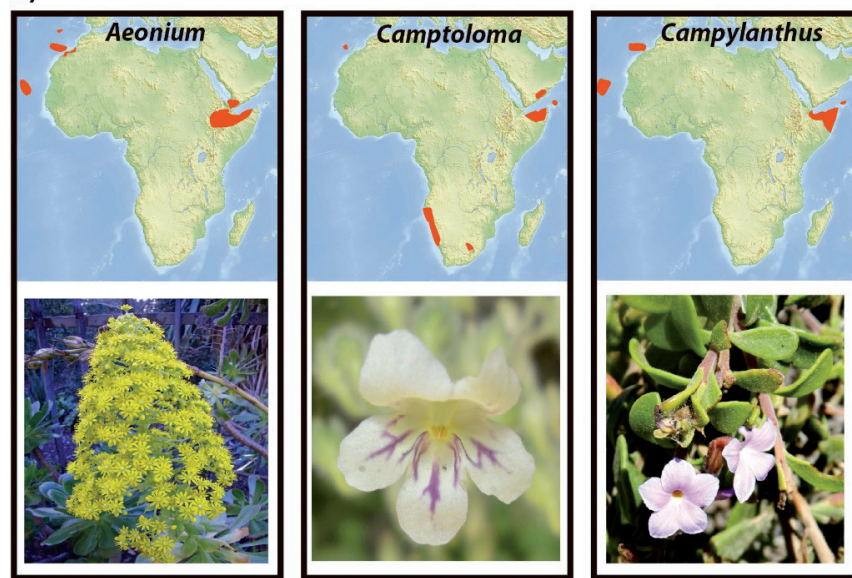

Figura 1.

a) Accidentes geográficos nombrados en el texto y de interés en la historia evolutiva de las floras del norte de África.

b) Ejemplos de géneros cuyas áreas de distribución (en naranja) muestran una disyunción geográfica con refugios a ambos lados del norte de África.

se encuentran situados a miles de kilómetros al este, en lugares tan distantes como el Cuerno de África y el archipiélago de Socotra (patrones conocidos como disyunciones biogeográficas: Figura 1b). Uno de los vestigios más notables de esta gran aridificación de África son los reducidos bosques subtropicales que quedan en ambos extremos del continente africano. Se considera que estos bosques aún albergan los últimos retazos de esa vegetación subtropical que sobrevivió a esta larga historia de extinción. Por tanto, debido a que proporcionan refugio a una biodiversidad muy antigua — primeras especies en migrar-y a su vez, muy amenazada, deben ser considerados santuarios de biodiversidad.

Un nexo evidente entre estos bosques son las campanillas silvestres del género Canarina (Figura 2). Aunque este género llegó a extenderse a lo largo del norte de África, al comen- zar la gran aridificación los ancestros de Canarina también quedaron confinados a ambos lados del Sáhara, y reducidos a tres especies que todavía sobreviven en refugios climáti$\cos$ (Figuras 1 b y 2): una de ellas -Canarina canariensis- al oeste del continente, refugiada en las laurisilvas y fayal-brezal de las Islas Canarias, mientras que otras dos especies $-C$. eminii y $C$. abyssinica- se encuentran restringidas a los bosques Afromontanos en el este de África (desde Etiopía hasta Malawi). Estamos, por tanto, ante un ejemplo de especies que se cobijaron en refugios climáticos característicos tanto de islas oceánicas como de "islas continentales" - hábitats de altura aislados por tierras bajas (Mairal et al. 2015a)_. Esta distribución produce un notorio grado de aislamiento ecológico y geográfico: mientras que los bosques Afromontanos están separados por hábitats de sabana, las laurisilvas canarias lo están por las barreras oceánicas entre islas (Figura 2). A su vez, la antigüedad de este aislamiento ha dado lugar a una gran diversidad genética entre las diferentes poblaciones de las tres especies del género Canarina (Mairal et al. 2015b, 2017b). Es muy importante conservar esta diversidad genética intraespecífica, ya que este es el nivel fundamental de la biodiversidad, que proporciona la base para el cambio evolutivo, y por lo tanto es crucial para mantener la capacidad de las especies para adaptarse a las nuevas condiciones ambientales.

Nuestro conocimiento sobre la historia evolutiva de los bosques subtropicales Afro-Macaronésicos indica que el declive gradual que sufrieron durante millones de años parece haberse incrementado alarmantemente en la época reciente. Estudios e informes conservacionistas han alertado sobre la posible contracción reciente de los últimos bosques Afro-Macaronésicos, con algunas cifras perturbadoras como que en Gran Canaria y en Tenerife la distribución potencial de la laurisilva ha quedado reducida a un 1\% y 10\% respectivamente (Santos-Guerra 1990; del Arco et al. 2010). Si el declive de estos bosques se ha intensificado debido a fenómenos recientes, como actividades humanas o cambio climático, se debería observar una señal genética vinculada a una reciente disminución demográfica; a la inversa, esta señal no aparecerá si los declives demográficos son más antiguos. Con el objetivo de testar esta hipótesis en el género Canarina, muestreamos poblaciones de $C$. canariensis y $C$. eminii a lo largo de su distribución geográfica (Figura 2). Posteriormente, utilizamos marcadores moleculares (microsatélites) y modelos demográficos y de modelización de nicho, para estimar los niveles de diversidad genética e inferir la evolución demográfica de las poblaciones.

Nuestros estudios demográficos mostraron que todas las poblaciones de Canarina han sufrido un declive poblacional reciente entre los últimos 10000-50000 años. A su vez, los modelos de distribución de especies mostraron que esto ha 
podido ser agravado por la pérdida de hábitat a causa del cambio climático, y probablemente por recientes actividades humanas como talas y quemas indiscriminadas y el pastoreo. Aunque las poblaciones de Canarina mostraron una alta estructura genética entre poblaciones — síntoma de su singularidad por una larga historia de aislamiento-, la diversidad genética dentro de las poblaciones fue muy baja — síntoma del declive demográfico en este contexto-. Esta situación podría provocar que las poblaciones sean más susceptibles a fenómenos como la endogamia o la estocasticidad demográfica.

De manera interesante, observamos que las poblaciones situadas en áreas más fragmentadas y dinámicas geológicamente sufrieron un declive mucho más acusado que las poblaciones situadas en áreas que han servido como refugio durante eventos volcánicos y oscilaciones climáticas, donde el declive poblacional detectado fue menor. Tal sería el caso de las paleo-islas en Canarias (Anaga, Teno, Roque del Conde) y el Monte Elgon en el este de África. Así, el carácter estático de estas áreas ha promovido que actúen como reservorios de una diversidad filogenética única, pero a su vez también como cunas y fuentes de diversidad hacia otras áreas. De esta manera, estas áreas deberían tener una conservación prioritaria, y deberían de ser gestionadas independientemente para salvaguardar la variabilidad genética de estas y otras especies.

En este estudio concluimos que, a pesar de que los esfuerzos conservacionistas suelen centrarse en especies raras de distribución reducida, las especies ampliamente distribuidas también pueden estar sujetas a altos grados de amenaza. A pesar de que las actuales especies de Canarina se encuentran todavía ampliamente distribuidas, sus poblaciones parecen estar sometidas a una "deuda de extinción". Esta deuda consiste en el retraso existente entre la pérdida de hábitat y el colapso total de las poblaciones, por lo que, aunque los efectos no son visibles a corto plazo, se manifestarán tras varias generaciones. Todo esto se verá agravado con la actual crisis climática y con las tasas alarmantes de crecimiento urbano de manera que, aunque este estudio se limitó a dos especies, esperaríamos detectar patrones similares en otras plantas. La deuda de extinción pronosticada, así como la espiral de extinción a las que estas poblaciones parecen estar sometidas, demandan medidas urgentes de conservación para proteger esta biodiversidad tan exclusiva, que convierte a estos ecosistemas en áreas de interés prioritario. A su vez, nuestro estudio hace hincapié en que es indispensable integrar la historia evolutiva de las especies para definir medidas de conservación más efectivas.

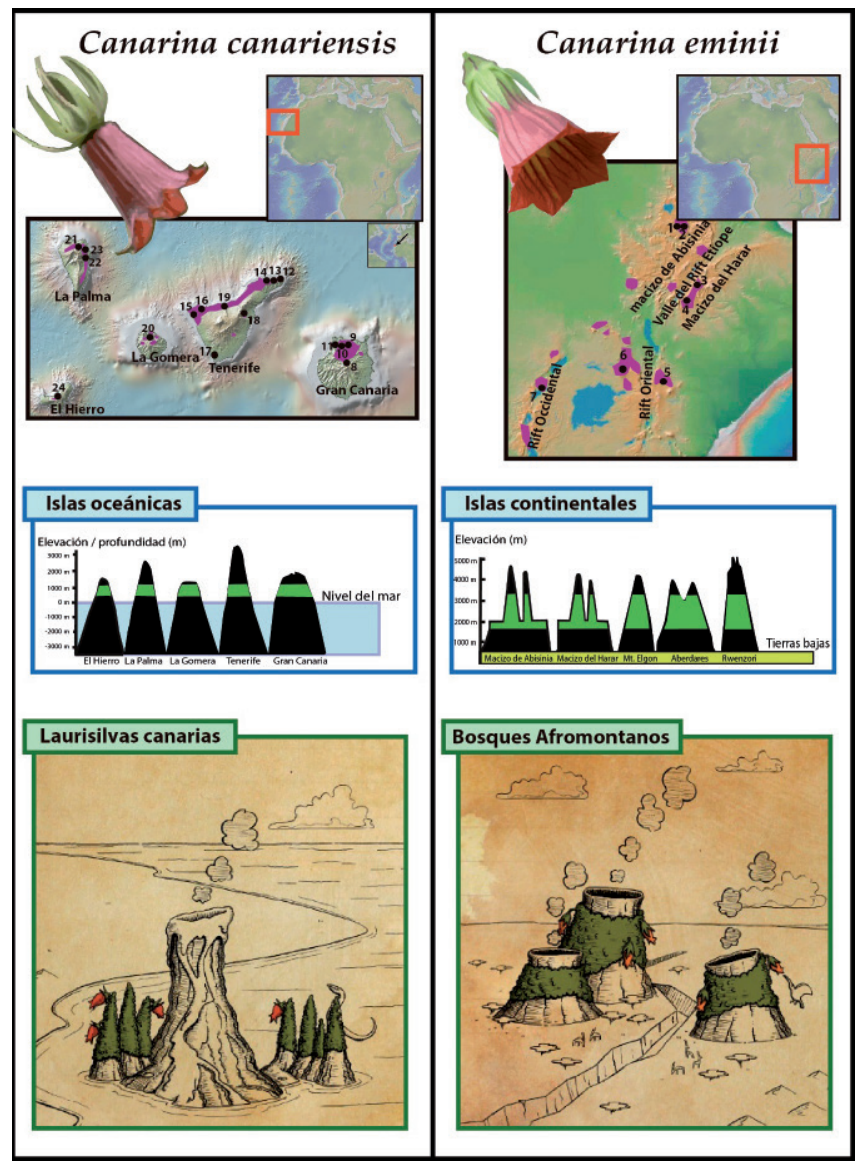

Figura 2. En la parte superior se muestra el área geográfica y distribución (en violeta) de las dos especies estudiadas: $C$. canariensis y C. eminii. Cada población muestreada se indica con un punto negro y un número, que se corresponde con los códigos incluidos en la Tabla 1 de la publicación original. En la parte central se muestran esquemas de los sistemas insulares y los cinturones de vegetación (en verde) ocupados por C. canariensis (laurisilvas canarias) y C. eminii (bosques Afromontanos). En la parte inferior izquierda se muestra una representación artística de los bosques de laurisilva en las paleo-islas de Tenerife (Teno y Anaga); y a la derecha una representación de los bosques Afromontanos separados por el Gran Valle del Rift. Figura adaptada de la publicación original (Mairal et al. 2018).

\section{MARIO $\begin{aligned} & \text { Stellenbosch University, Department of Botany } \\ & \text { and Zoology, Natural Sciences Building. }\end{aligned}$ E-mail: mariomairal@gmail.com; www.mariomairal.com}

\section{Agradecimientos}

A Isabel Sanmartín por su apoyo para escuchar, enriquecer y discutir esta investigación. Este trabajo no habría podido llevarse a cabo sin las estancias de investigación e ideas que me facilitaron: Juli Caujapé Castells y Ruth Jaén Molina en el Jardín Botánico Canario "Viera y Clavijo"-UA CSIC, Nadir Álvarez en la Universidad de Lausanne (Suiza), Myriam Heuertz y Loïc Pellissier en la Universidad de Freiburg (Suiza). Agradezco una lectura crítica de este artículo a Juli Caujapé Castells y Ruth Jaén Molina. A la junta directiva de la SEBICoP por la concesión del Premio César Gómez Campo.

\section{Bibliografía}

- Axelrod, D.I. \& P.H. Raven (1978). Late Cretaceous and Tertiary vegetation history of Africa. In: Werger, M.J.A. (ed.) Biogeography and Ecology of Southern Africa. Springer, the Netherlands, pp. Southern

- del Arco Aguilar, M.J., R. González-González, V. Garzón-Machado \& B. Pizarro- Hernández (2010) Actual and potential natural vegetation on the $\mathrm{Ca}$ nary Islands and its conservation status. Biodiversity and Conservation 19: 3089-3140.

- Mairal, M., L. Pokorny, J.J. Aldasoro, M. Alarcón \& I. Sanmartín (2015a). Ancient vicariance and climate-driven extinction explain continental-wide disjunctions in Africa: the case of the Rand Flora genus Canarina (Campanulaceae). Molecular Ecology 24: 1335-1354.
- Mairal, M., I. Sanmartín, J.J. Aldasoro, V. Culshaw, I. Manolopoulou \& M. Alarcón (2015b). Paleo-islands as refugia and sources of genetic diversity within volcanic archipelagos: the case of the wiwithin volcanic archipelagos: the case of the Wilaceae). Molecular Ecology 24: 3944-3963.

- Mairal, M., I. Sanmartín \& L. Pellissier (2017a). Lineage-specific climatic niche drives the tempo of vicariance in the Rand Flora. Journal of Biogeography 44: 911-923.

- Mairal, M., I. Sanmartín, A. Herrero, L. Pokorny, P. Vargas, J.J. Aldasoro \& M. Alarcón (2017b). Geo graphic barriers and Pleistocene climate change shaped patterns of genetic variation in the Eastern Afromontane biodiversity hotspot. Scientific Reports 7: e45749.
- Santos-Guerra, A.S. (1990). Bosques de laurisilva en la región macaronésica. Serie Naturaleza y Medio Ambiente, No. 49. Strasbourg: Council of Europe. 